deren installiert und personalisiert der TSM über das Mobilfunknetz die Bezahlapplikation auf die SIM-Karte.

Bei Verlust des Smartphones kann der Kunde die Sperrung der Wallet veranlassen. Die Bezahlapplikation wird dann sofort von G\&D als TSM über das Mobilfunknetz gelöscht. Gleichzeitig wird die Karte im Hintergrundsystem der Bank gesperrt wie es heute auch bei Bank- und Kreditkarten üblich ist. "Gemeinsam mit G\&D erfüllen wir das Sicherheitsbedürfnis der Nutzer. Aber auch bei der Anwendungsvielfalt setzen wir einen hohen Standard. Unsere Mobilfunkkunden können vom Start weg das sichere Bezahlen über die Mobile Wallet mit dem Einlösen digitaler Coupons kombinieren. Auch Mitgliedsausweise wie die ADAC-Karte lassen sich im Smartphone speichern. Das Portemonnaie muss ab jetzt nicht mehr immer dabei sein, während das zur digitalen Brieftasche ausgebaute Smartphone weiter an Bedeutung für den Alltag der Kunden gewinnen wird", sagt Martin Deventer, Director Core Network and Services bei E-Plus.

\section{LfD RLP: Facebook manipuliert Nachrichtenströme}

Anfang Juli 2014 ist bekannt geworden, dass Facebook im Jahr 2012 fast 700000 (englischsprachige) Nutzerinnen und Nutzer bewusst dadurch manipuliert hat, dass es seine Nachrichten nach den Kriterien "positiv" und "negativ" ausgewählt und gesteuert hat. Ziel war es, herauszufinden, ob durch eine solche Selektion die Reaktionen der Nutzerinnen und Nutzer beeinflusst werden können, ob also die Posts der Nutzerinnen und Nutzer in Reaktion auf die Auswahl der ihnen zugesandten Nachrichten ebenfalls eher negativ oder positiv ausfallen. Dies erinnert an Experimente von Verhaltensforschern mit Laborratten.

Bemerkenswert an diesem Vorgehen von Facebook sind mehrere Punkte:

- Facebook sieht es als sein selbstverständliches Recht an, den Nachrichtenstrom, den es an seine Nutzerinnen und Nutzer leitet, an seinen Bedürfnissen auszurichten. Ein Recht der Nutzerinnen und Nutzer auf (möglichst) objektive und umfassende Information wird nicht anerkannt.

- Es ist ein wichtiges Ziel von Facebook, in das es in nennenswertem Umfang Mittel investiert, herauszufinden, wie seine Nutzerinnen und Nutzer manipuliert werden können. Darin liegt der eigentliche Skandal: Nutzerinnen und Nutzer werden nicht als Individuen mit eigenen Rechten betrachtet, sondern als Masse, die Facebook nach Kräften so steuern darf, wie es in sein Geschäftsmodell und in seine Interessen passt. Bei der monopolähnlichen Stellung von Facebook mit fast einer Milliarde Nutzerinnen und Nutzern ist eine solche Haltung besonders gefährlich.

Dessen sollten sich alle diejenigen bewusst sein, die Facebook einsetzen und nutzen. Facebook ist kein neutraler Dienstleister, der nur den Austausch von Informationen zwischen seinen Nutzerinnen und Nutzern erleichtern und ihnen so das Leben bequemer machen will. Facebook ist ein Unternehmen, das vor der gezielten Beeinflussung der Nachrichten nicht zurückschreckt.

\section{Deutscher Anwaltstag: Privatheit zwischen Schutzgut und digitaler Währung}

Ein Forum für eine hochkarätige und überaus spannende Diskussion bot der 65. Deutsche Anwaltstag in Stuttgart (26. bis 28. Juni 2014) mit der Schwerpunktveranstaltung „Freiheit gestalten! - Privatheit zwischen Schutzgut und digitaler Währung". Die Diskutanten veranschaulichten vor über 200 Teilnehmern das Spannungsverhältnis zwischen dem kostenfreien Nutzen digitaler Dienstleistungen im Internet einerseits und dem Erhalt digitaler Freiheit andererseits.

Harald Lemke, Vorsitzender von Selbstregulierung Informationswirtschaft, wies auf das Paradoxon hin, dass viele Menschen zwar anscheinend nicht wollten, dass Unternehmen aufgrund ihrer Datennutzung Profile erstellten und kommerziell verwerteten - die entsprechenden Internetdienste aber dennoch intensiv nutzten. „Eine Datenschutzregelung im deutschen Recht hilft auch angesichts der global verlaufenden Datenströme nicht weiter", sagte Lemke, „wir werben für "Ko-Regulierung ", mit der Wirtschaft".

Der Hacking-Experte Sebastian Schreiber verortete das Problem in der Intransparenz des Internets für die Nutzer: „Wenn der Internetservice kostenfrei ist, ist der Mensch die Ware und kein Kunde". Zu dem, was die Wirtschaft auch nach dem Google-Urteil des EuGH über die Internetnutzer wissen könne, sagte Schreiber: „Ein endgültiges Löschen meiner Daten ist gar nicht möglich bei den aktuellen Servern. Es wäre schon viel gewonnen, wenn die Nutzer viel mehr auf Verschlüsselungen setzen würden, um das Sicherheitsniveau zu erhöhen."

„Wir brauchen höhere Datenschutzstandards für alle. Deshalb brauchen wir die Datenschutz-Grundverordnung", erklärte der oberste irische Datenschutzbeauftragte Billy Hawkes. „Damit das geltende Datenschutzrecht respektiert wird, müssen die Datenschutzbehörden in der Praxis mehr kooperieren", forderte Hawkes, der für die Überwachung vieler amerikanischer Internetunternehmen zuständig ist, die in Irland ihren europäischen Sitz haben.

Paul Nemitz, in der EU-Kommission verantwortlich für die Grundrechte, kritisierte, dass Deutschland bei der europäischen Regulierung des Datenschutzes im öffentlichen Bereich mehr „Flexibilität" fordere. „Und das zu einem Zeitpunkt, in dem die Öffentlichkeit zunehmend über die Aktivitäten der Nachrichtendienste erfährt", sagte Nemitz. Er warnte davor, die Verhandlungen über eine europäische Datenschutzregelung mit zusätzlichen Forderungen zu belasten. „Ich wünsche mir, dass die Bundesregierung jetzt wie angekündigt handelt und den Abschluss der Datenschutz-Grundverordnung unterstützt", so der ranghohe Kommissionsbeamte.

Die Podiumsdiskussion wurde moderiert durch Rechtsanwalt Prof. Dr. Peter Bräutigam, dem Vorsitzenden des Beirats der Stiftung Datenschutz.

\section{Steigende Userzahlen: Ein TOR, wer Privatsphäre dabei denkt}

Ein Kommentar von Sicherheitsspezialist Udo Schneider, Pressesprecher beim IT-Sicherheitsanbieter Trend Micro

Viel und aufgeregt wird zurzeit über TOR diskutiert. Doch das äußerst effektive Anonymisierungsnetzwerk wird nicht nur bei den Verfechtern der Privatsphäre im Internet immer beliebter, deren Hoffnungen freilich aufgrund der jüngsten Enthüllungen zu den 